\title{
ESTUDIO DE LAS NORMAS INTERNACIONALES DE INFORMACIÓN FINANCIERA APLICADAS A LA PRESENTACIÓN DE ESTADOS FINANCIEROS
}

\author{
Study of International Financial Reporting Standards applied to the presentation of \\ financial statement
}

Laura Alejandra Diaz Amado

ladiaz@poligran.edu.co

Institución Universitaria Politécnico Grancolombiano

Contaduría Publica

Colombia

Resumen

El estudio de las normas internacionales aborda la historia de las instituciones emisoras desde el International Accounting Standards Committee (IASC) y el International Accounting Standards Board (IASB) que es el organismo que sustituye al IASC para implementar las NIIF y modificar las NIC. Así mismo, se evidencia que el marco conceptual se denomina en un contexto global la constitución contable y financiera que da conocimiento a las definiciones, las características y otros conceptos fundamentales para conocer y determinar la aplicación de las normas en la información financiera y contable; por lo que es necesario evidenciar los procesos de convergencia de los países pertenecientes a la Alianza del Pacífico que tienen un acercamiento político, económico, social y ambiental con Colombia, que da pie a estudiar el sector textil en Colombia y su dinamismo en la economía, conociendo que es uno de los más importantes en el Producto Interno Bruto y que exporta a más de 1.500 empresas. Así se logra evidenciar las compañías de mayor relevancia en el sector y subsector confección, para seleccionar la muestra de empresas que enmarcan el funcionamiento de las normas, por medio de un diagnóstico financiero y un análisis a sus estados financieros bajos las normas internacionales de información financiera y contable.

Palabras clave:

1. Normas internacionales

2. Sector textil

3. Diagnóstico financiero 


\section{INTRODUCCIÓN}

El estudio de las normas internacionales de información financiera se debe generar conociendo la creación de ellas a partir de los inicios de las instituciones emisoras de las normas. Por esto, es necesario contextualizar el inicio del International Accounting Standars Committee que se genera por la necesidad de agrupar aquella información contable que se convierte en un factor importante para la toma de decisiones en un mundo globalizado donde las empresas manejan un mercado de capitales. La organización para la cooperación y el desarrollo económico y las Naciones Unidas han sido de gran importancia, en especial en los años 80’ donde enfrentaban la crisis económica de los países potencia, posguerras y la guerra fría; y que así mismo, generaron opiniones y críticas sobre el funcionamiento de estas normas, que generó descontento y obligó la restructuración de las normas; por lo que se expone la implementación de las NIIF en el marco global.

Como parte del marco teórico se evaluará qué es el marco conceptual y la Norma Internacional de Contabilidad 1 que son los pilares para el funcionamiento económico y financiero de una empresa. Así mismo, se realizará una contextualización de los procesos de convergencia de los países que hacen parte de la Alianza del Pacifico; para así denotar y comparar como se implementaron y los impactos económicos.

La economía colombiana cuenta con diferentes sectores que enmarcan las finanzas del país; donde se evidencia que uno de los sectores con mayor dinamismo es el textil. Razón por la cual se generó la siguiente pregunta problema ¿Cómo las empresas del sector textil están aplicando las cualidades de la información financiera en la presentación de sus Estados Financieros?

Para dar respuesta al problema de investigación se realizará el diagnóstico financiero y el análisis de los estados financieros y las notas contables de la empresa. Por esta razón se considera que este será un estudio de investigación cuantitativo que permitirá dar a conocer si la información que presenta la información es útil en cifras numéricas para la toma de decisiones y para evidenciar la rentabilidad, endeudamiento y liquidez de la muestra de empresas. Así mismo, se evaluarán aquellos aspectos que definen porque es cuantitativa y la razón de la investigación del análisis de los estados financieros (Peña \& Herrera, 2016).

Se considera como materia importante de la investigación explicar y determinar que sucede con estas empresas para así comprender si el uso de las normas internacionales es el adecuado. Dentro de la investigación se tomarán datos de fuentes como EMIS, el IFRS, entre otras que ayudarán a sustanciar la investigación.

Esta investigación permitirá aumentar el conocimiento sobre la relación de la contabilidad y las finanzas acogidas en un marco internacional que tiene como fin globalizar el mercado. Así mismo, presentará las evidencias de cómo funciona el sector textil en Colombia y porque se elige para el estudio de este trabajo y poder determinar los diferentes indicadores financieros que determinan cómo funcionan las empresas y si sus ganancias son útiles para los usuarios.

\section{Método}

La implementación de las NIIF ha generado grandes impactos en la economía mundial, como se ha evidenciado en América Latina; lo que ocasionó que en los últimos años se presentaran varios estudios que evalúan tanto a las normas internacionales como al diagnóstico financiero de las empresas.

Para entender mejor estos impactos y en especial en lo que concierne al diagnóstico financiero de empresas, con estados preparados bajo NIIF, se abarca en el marco teórico del proyecto, los antecedentes del emisor de normas IASB, la concepción epistemológica del marco conceptual de las NIIF y los procesos de convergencia de la Alianza del Pacífico (Parra-Mayorga, 2019). 
De esta manera, se contextualiza el sector textil en Colombia que cuenta con un $6 \%$ de representación en el Producto Interno Bruto industrial y que maneja 1.333 empresas que actualmente exportan a mas de 107 países en el mundo.

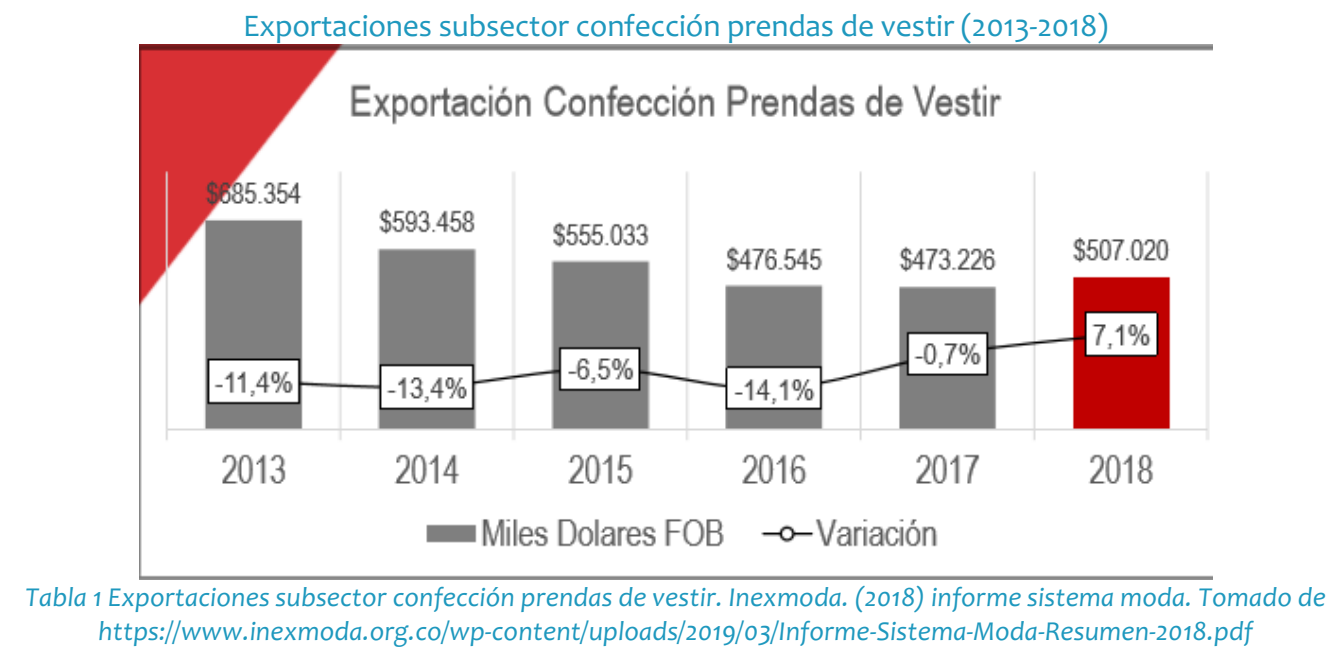

La tabla 1 permite evidenciar que el año 2018 tuvo una mejor cara para el sector que el 2017, dada la reactivación de la economía y los beneficios que ha buscado el gobierno para persuadir aquella competencia externa como es la China y de Bangladesh. Su posicionamiento en el mercado se debe a la calidad, la mano de obra y sus diseños exclusivos; lo que permite que sea una plataforma ideal para exportar y ampliar el campo de negocios en América Latina. "actualmente se producen y exportan productos como jeans, ropa interior femenina y masculina, ropa de hogar, fajas y ropa de control, ropa formal, suéteres, vestidos de baño femeninos y masculinos, entre otros." (PROCOLOMBIA, 2017).

Para dar respuesta al problema abordado se realizada un estudio de una muestra de empresas del sector textil en Colombia; por medio de, una investigación cuantitativa en la que se parte de la recolección de datos de la muestra en un periodo de cinco años (2014-2018); para así desarrollar el diagnóstico financiero con los tres aspectos financieros de rentabilidad, endeudamiento y liquidez (Elisondo \& Donolo, 2015; García, 2015; Norman-Acevedo, 2019).

Este estudio tiene un alcance descriptivo dado que la etapa de recolección de datos se realiza a las tres empresas elegidas del sector textil en un periodo de tiempo específico, escogiendo los estados financieros con mayor relevancia para la toma de decisiones; así poder definir y especificar la importancia del uso de la NIC 1 y las características cualitativas de la información financiera. Así mismo, cuenta con un diseño no experimental por su manejo de variables que no manipulan la información si no que se analiza por la situación o la circunstancia.

\section{Resultados}

Después de revisar el surgimiento de las normas internacionales y los aspectos fundamentales del marco conceptual y la NIC 1 fue necesario analizar como el sector textil implementa las normas internacionales; por esta razón, se toma una muestra de 3 empresas que son seleccionadas de acuerdo con su importancia en el sector y el grupo en el que se encuentran, escogiendo a Industrias Cannon de Colombia S.A., Coltejer S.A. y Fabricato S.A. que pertenecen al grupo I NIIF plenas.

Se evidenció que las dos empresas Coltejer S.A. y Fabricato S.A. permitieron identificar sus informes financieros compuestos por sus estados financieros y las políticas contables que denotan los cumplimientos de las cualidades y características especificadas en el marco conceptual, esto correspondiente a cada periodo; mientras que, Industrias Cannon de Colombia S.A. no permitió 
analizar sus informes financieros con las notas respectivas dado que solo cuentan con datos numéricos que se presentan en bases de datos como EMIS Benchmarking.

Es importante precisar que por medio de esta información se pudo denotar que al estudiar las variables financieras estos generarían resultados acertados y confiables. El índice de rentabilidad permitió demostrar que las empresas del sector textil tuvieron una decaída a partir del 2016; considerando que estas tres compañías son las representantes de la economía textil y su margen de rentabilidad no ha sido el óptimo para los últimos años por situaciones como la competencia extranjera, aquellas tasas aduaneras elevadas y la economía colombiana que enfrentaba una crisis.

Se analiza el endeudamiento para denotar como ha funcionado el sector y cuál es la más financiable que se determina con la que contenga un menor endeudamiento. Fabricato S.A. e Industrias Cannon de Colombia S.A. cuentan con un endeudamiento total bajo y que en el último año disminuyó lo que permite demostrar que son las más financiables dentro de un sector que se encuentra en su peor etapa.

Dentro de estos indicadores es esencial denotar la liquidez de las empresas donde se analizó el capital de trabajo neto operativo que demuestra que Coltejer ha tenido una decaída demostrando su falta de liquidez monetaria para financiar sus obligaciones; por otro lado, se evidencia que Fabricato tiene una mayor capacidad de cumplir con sus obligaciones monetaria e Industrias Cannon se ha encontrado en un limbo más estable que los otros dos entes.

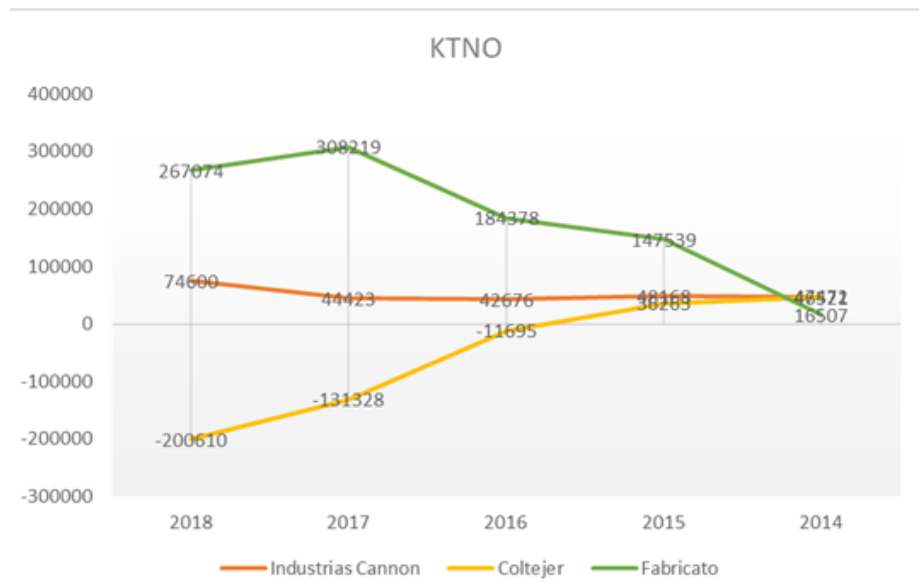

Figura 1. Capital de trabajo neto operativo. Elaboración propia a partir de datos extraídos de EMIS Benchmarking.

Al finalizar es importante resaltar que estas tres compañías manejan sus estados financieros bajo las Normas Internacionales de Información Financiera y Contable; donde cumple con los estados financieros que estipula la NIC 1, el estado de situación financiera, el estado de resultados, el estado de cambios en el patrimonio, el estado de flujos de efectivo y las notas contables; así mismo, Coltejer y Fabricato especifican en sus políticas contables que presentan su información bajo las características cualitativas de la información financiera, esto quiere decir que es fiable, con representación fiel, relevancia, entre otras.

\section{Discusión y Conclusión}

Después de la investigación realizada se puede concluir que las Normas Internacionales de Contabilidad y de Información Financiera se crearon con el fin de disminuir las diferencias de las prácticas contables y financieras en los países con mercados de capitales. 
Finalmente se determina que el sector textil representa el 6\% del PIB industrial por su calidad, mano de obra y diseños exclusivos; así mismo, se considera una de las principales plataformas para exportar y ampliar el campo de negocios. De manera que, se evaluó la inclusión de las normas internacionales en los países pertenecientes a la Alianza del Pacifico; en contraste con esto, Colombia fue el país que se retardó para implementar las normas internacionales hasta el año 2015 (Hernández, Wilches, \& Robles, 2015; Obando, 2015; Rigo \& Donolo, 2016).

Así mismo, se logró evidenciar que la muestra de empresas seleccionadas del sector es perteneciente al grupo I que se encuentran las grandes empresas con una alta suma de ingresos y de activos con la mayor antigüedad en el mercado, que así mismo las convierte en las de mejor calidad, innovación y diseño (Arias-Velandia, Rincón-Báez, \& Cruz-Pulido, 2018; Díaz, 2016; Mendoza, 2016). El diagnóstico financiero evidencia la situación del sector textil desde el 2016 al 2018, estas tres empresas son reconocidas a nivel mundial por sus productos y por su recorrido en el mercado de confección; que demuestra que la rentabilidad de las empresas especialmente de Fabricato ha disminuido.

Así mismo, se resalta que estas empresas dentro del contexto cumplen con la implementación de las normas internacionales y que el uso de estas en sus estados financieros permiten denotar a problemática financiera, y que la información es fiel, relevante y comparable como se denota en el diagnóstico realizado.

\section{Referencias bibliográficas}

Arévalo, L. G. (2014). La alianza Pacífico: geopolítica e integración económica. Revista Vía luris, págs. 159-172.

Arias-Velandia, N., Rincón-Báez, W. U., \& Cruz-Pulido, J. M. (2018). DESEMPEÑO DE MUJERES Y HOMBRES EN EDUCACIÓN SUPERIOR PRESENCIAL, VIRTUAL Y A DISTANCIA EN COLOMBIA - Women and men performance in face-to-face, virtual and distance higher education in Colombia. Panorama, 12(22), 57-69. https://doi.org/10.15765/pnrm.v12i22.1142

Díaz, A. M. (2016). Evaluación de la propuesta de enseñanza de las áreas de lenguaje y matemáticas en la Institución Educativa San José del Pantano. Panorama, 9(17), 25-39. https://doi.org/10.15765/pnrm.v9i17.789

Elisondo, R. C., \& Donolo, D. S. (2015). Creatividad y alfabetización informacional. El desafío en cuatro propuestas. Panorama, 8(15), 23-33. https://doi.org/10.15765/pnrm.v8i15.547

García, V. S. (2015). La gestión local como propuesta para la solución de problemas comunes de urbanismo en América Latina. Panorama, 9(16), 92-103. https://doi.org/10.15765/pnrm.v9i16.633

Hernández, N. M. F., Wilches, J. C. P., \& Robles, J. R. (2015). Desarrollo de los niveles de razonamiento geométrico según el modelo de Van Hiele y su relación con los estilos de aprendizaje. Panorama, 9(16), 44-54. https://doi.org/10.15765/pnrm.v9i16.635

IFRS. (2012). Instituto Nacional de Contadores Públicos. Obtenido de Instituto Nacional de Contadores Públicos: http://www.aplicaciones-mcit.gov.co/niif/niif.php?anio id=2012

Inexmoda. (2018). Informe sistema de moda. Obtenido de https://www.inexmoda.org.co/wp-content/uploads/2019/03/InformeSistema-Moda-Resumen-2018.pdf

Mendoza, J. A. G. (2016). Empresa privada: principal socio en el posconflicto y la construcción de la paz. Panorama, 10(18), 84-92. https://doi.org/10.15765/pnrm.v10i18.823

Nava, R., \& Marbelis, A. (2009). Análisis financiero: una herramienta clave para una gestión financiera eficiente. Revista Venezolana de Gerencia, 606-628.

Norman-Acevedo, E. (2019). NUEVOS LENGUAJES PARA APRENDIZAJE VIRTUAL HERRAMIENTAS PARA LOS ESCENARIOS DE APRENDIZAJE. Panorama, 13(24), 5-7. https://doi.org/10.15765/pnrm.v13i24.1214

Obando, C. R. C. (2015). Movimientos barriales: reivindicación del derecho a la ciudad. Panorama, 9(16), 66-76. https://doi.org/10.15765/pnrm.v9i16.640

Parra-Mayorga, R. A. (2019). INVESTIGACIÓN EN UNIVERSIDADES DE BOGOTÁ SOBRE EL CONOCIMIENTO Y DESTREZA DE LOS FUTUROS CREADORES DE CONTENIDO DIGITAL PARA LA CONSTRUCCIÓN DE FORMATOS DE ANUNCIO EN FACEBOOK. Panorama, 14(27), 89-108. https://doi.org/10.15765/pnrm.v14i27.1529

Peña, B. I. M., \& Herrera, L. K. D. (2016). Experiencia de cooperación en el acompañamiento empresarial integral en el territorio como aporte al proceso de reintegración en el posconflicto. Panorama, 10(18), 67-82. https://doi.org/10.15765/pnrm.v10i18.824

Pereda Tua, J. (S.f.). El marco conceptual para la información financiera. Monografías sobre las normas internacionales de información financiera, 137-285. Obtenido de El criterio.

Rigo, D. Y., \& Donolo, D. (2016). Evaluación... más de los mismo desafiando formatos y modalidades sin libreto. Panorama, 10(19), 62-75. https://doi.org/10.15765/pnrm.v10i19.829

Sampieri, R., \& Mendoza, C. (2018). Formulación de hipótesis en la ruta cuantitativa. En R. Sampieri, \& C. Mendoza, Metodología de la investigación: Las rutas cuantitativa, cualitativa y mixta (págs. 122-146). Ciudad de México: Mc Graw Hill Education. 